\title{
Exploration of the Mechanism and Active Targets of Chinese Herbal Formula, JTTZ, for the Treatment of Type 2 Diabetes with Obesity and Hyperlipidemia Based on Network Pharmacology
}

\author{
Haiyu Zhang ( $\sim$ zhy20130322015@sina.com ) \\ China Academy of Chinese Medical Sciences Guanganmen Hospital \\ Xuedong An \\ China Academy of Chinese Medical Sciences Guanganmen Hospital \\ De Jin \\ China Academy of Chinese Medical Sciences Guanganmen Hospital \\ Jiaxing Tian \\ China Academy of Chinese Medical Sciences Guanganmen Hospital \\ Wenke Liu \\ China Academy of Chinese Medical Sciences Guanganmen Hospital \\ Shenghui Zhao \\ China Academy of Chinese Medical Sciences Guanganmen Hospital \\ Xinmiao Wang \\ China Academy of Chinese Medical Sciences Guanganmen Hospital \\ Yeying Hu \\ Beijing University of Chinese Medicine \\ Fengmei Lian \\ China Academy of Chinese Medical Sciences Guanganmen Hospital \\ Xiaolin Tong \\ China Academy of Chinese Medical Sciences Guanganmen Hospital
}

\section{Research}

Keywords: Jiangtang Tiaozhi (JTTZ) formula, Type 2 diabetes (T2D), Network pharmacology, Adipose tissue dysfunction, Inflammation, Insulin resistance

Posted Date: January 20th, 2021

DOI: https://doi.org/10.21203/rs.3.rs-147873/v1

License: (-) (1) This work is licensed under a Creative Commons Attribution 4.0 International License. Read Full License 


\section{Abstract}

Background

Previous studies have indicated that the JTTZ formula exhibits clinical benefit in T2D with obesity and hyperlipidemia such as lowering blood glucose, blood lipids, weight, and ameliorating symptoms as well as regulating islet function. However, their mechanism of action remains unclear. T2D with obesity and hyperlipidemia is associated with a severely poor management duo to difficulty in achieving the clinical goals and lack of effective multi-targeted therapies. In this study, we explored its potential mechanisms and therapeutic targets by network pharmacology.

\section{Methods}

The active ingredients and targets of JTTZ were obtained in the TCMSP, TCMID, TCM Database@Taiwan, PubChem and Swiss Target Prediction. And the therapeutic targets were searched from TTD, DrugBank Database and DisGeNET. Then, topology analysis were used as secondary screens to identify key hubs of the network. Finally, the data was integrated by Cytoscape software to construct a common network module. PPI networks were visualized to identify the interaction of the candidate targets. GO and KEGG pathway analysis were implemented. Rerult: 110 active compounds and 166 candidate targets of JTTZ against T2D with obesity and hyperlipidemia were obtained to construct compound-targets network. And, the therapeutic targets AKT2, RELA, NFKB1 and GSK3B were identified. GO and KEGG pathway analysis indicated that the biological processes related to inflammatory response, insulin secretion, steroid and bile acid metabolism, and 13 pathways mainly including adipocytokine signaling pathway, cAMP signaling pathway and cGMP-PKG signaling pathway were enriched.

\section{Conclusion}

Our data established that JTTZ intervenes with adipose tissue dysfunction via regulating to the adipocytokine (leptin and adiponectin), AMPK signaling pathway, cAMP and cGMP-PKG signaling pathway, inhibits systematic inflammatory response by NF-KB and MAPK signaling pathway, and ameliorates insulin resistance through PI3K/AKT2 pathway, all of which could thus offer a promising therapeutic strategy. In addition, AKT2, RELA, NFKB1 and GSK3B were identified to be regarded as potential therapeutic targets as well.

\section{Introduction}

The prevalence of type 2 diabetes (T2D) and obesity has continued to increase in over five decades, which causes major threats to human health globally (13). In 2019, it was estimated that 463 million people live with diabetes, in which T2D was account for $90 \%$ of all diabetes, and it was predicted to double by $2045(4,5) .60 \%$ of patients with T2D are obese (body mass index, BMI $\geq 30 \mathrm{~kg} / \mathrm{m} 2$ ) , and $70-80 \%$ have hyperlipidemia(5, 6). Insulin resistance in liver, skeletal muscle, and adipose tissue accompanied by dysfunction of pancreatic islet $\beta$ cells is the common underlying abnormality of T2D and obesity and hyperlipidemia. However, causes of T2D and obesity remain largely unclear $(2,6)$. At present, metformin is the first-line drug for the treatment of T2D by reduced hepatic glucose output and improvement of obesity-induced metabolic inflammation(7). Although there is several well-established drug classes for treating T2D, it is still a considerable unmet need for a drug that reverses diseases progression base on combined treatment strategies(8).

Our previous studies showed that JTTZ fomula, the Chinese herbal formula, could safely and significantly decrease the blood glucose, blood lipids, and body weight, enhance islet $\beta$ cell function, improve insulin resistance for T2D patients with obesity and hyperlipidemia, which has been explored via a multicenter randomized, positive-controlled clinical trial with a total of 450 participants(9). The JTTZ consists of eight herbs, including Radix Salviae, Zingiberis Rhizoma, red yeast rice, Coptidis Rhizoma, Momordica charantia, Aloe vera, Schisandrae Chinensis Fructus and Anemarrhenae Rhizoma, which is derived from a classic formula, Dahuang Huanglian Xiexin Decoction. These herbs achieves effects on improvement of glucose and lipid metabolic disorder by interaction of different biological functions. Although we had confirmed the significant therapeutic effect of JTTZ against T2D with obesity and hyperlipidemia by clinical trials(9), its action mechanism and molecular targets is still unclear, which would limit it as a potential drug to be widely used in clinical practice.

The network pharmacology adopt a network perspective to describe the complex interactions among genes related to diseases and drugs, which is in accord with the holistic theory of traditional Chinese medicine (TCM)(10-12). There had been so many studies for revealing the potential mechanism and action targets of classic formula by applying TCM network pharmacology methods(13-15). It is appropriate for the TCM network pharmacology methods to reveal drug-gene-disease associations, so we explored the possible mechanism and potential therapeutic targets of JTTZ against T2D with obesity and hyperlipidemia through TCM network pharmacology. The flowchart of the experimental procedures of our study is shown in Fig. 1.

\section{Materials And Methods}

\section{Active Ingredients Screening and Database Building}

The chemical components of JTTZ were identified from the Traditional Chinese Medicine Systems Pharmacology Database (TCMSP, Version 2.3. http://Isp.nwu.edu.cn/tcmsp.php/), Traditional Chinese Medicine Integrative Database (Version 2.03. http://www.megabionet.org/tcmid/) and TCM Database@Taiwan (http://tcm.cmu.edu.tw/).TCMSP is a database of systems pharmacology for drug discovery by revealing the mechanisms of Chinese herbal medicines(16). TCMID is a comprehensive database to provide information and bridge the gap between Traditional Chinese Medicine and modern life sciences(17). TCM Database@Taiwan is currently the largest non-commercial TCM database for drug screening(18). We set the indexes of oral bioavailability $(\mathrm{OB}) \geq 30 \%$ and drug-likeness $(\mathrm{DL}) \geq 0.18$ as screening criteria. The candidate ingredients which satisfies the selective threshold of $\mathrm{OB}$ and $\mathrm{DL}$ were regarded as the potential component. 


\section{The Identification of Potential Targets of the Ingredients}

All the candidate components were retrieved in PubChem (https://pubchem.ncbi.nlm.nih.gov/) to obtain Simplified Molecular-Input Line-Entry System (SMILES) that is computed from chemical structure devoid of isotopic and stereochemical information. PubChem is the largest online platform for collection of chemical information from authoritative sources. At the same time, the validated targets were extracted from PubChem. In addition, the predicted targets were obtained by importing SMILES in Swiss Target Prediction (http://www.swisstargetprediction.ch/), a web server for predict the targets of bioactive molecules base on a combination of 2D and 3D similarity(19). Then, we unified target-name using the Retrieve/ID mapping tool in UniProt (http://www.uniprot.org/), which could convert protein into official symbol formats of gene targets(20).

\section{The Identification of Known Therapeutic Targets}

Therapeutic targets associated with T2D and obesity and hyperlipidemia were searched from Therapeutic Target Database (TTD, https://db.idrblab.org/ttd/), DrugBank Database (DB, version 5.1.5. https://www.drugbank.ca/), and DisGeNET (DGN, version 6.0. http://www.disgenet.org/). TTD is a database for facilitating discovery of therapeutic targets(21). DrugBank is a resource that combines detailed drug data with comprehensive drug target information (22). And DisGeNET is a platform containing multitudinous genes associated with human diseases(23). We searched for the keywords "type 2 diabetes" "obesity" and "hyperlipidemia" trough the above databases, respectively. After selecting targets that appear in all three diseases, we also unified target-name using the Retrieve/ID mapping tool in UniProt. Subsequently, therapeutic targets were compared with candidate gene targets of active ingredients to screen the potential targets of JTTZ related to T2D with obesity and hyperlipidemia.

\section{Network Construction}

The compounds-targets network was constructed and visualized in the Cytoscape software (version 3.7.2, Boston, MA, USA, https://cytoscape.org/)(24), which showed the relationship between the possible targets of JTTZ and the known targets of T2D with obesity and hyperlipidemia. The node shape, color and size were set in the Cytoscape software by generate style from statistic tool. The different node shape and color reflected different categories, such as targets, compounds and pathways, et al. The size of node depends on the magnitude of the degree centrality (DC) and betweenness centrality (BC), which reflects the number of direct links to nodes and describe the topological importance of targets in networks. The protein-protein interaction (PPI) network carried out by the string software (version 11.0, https://string-db.org/)(25), with species limited to "Homo sapiens", and results were saved in PNG and TSV format. The PNG files were showed as figure and the TSV files were imported into Cytoscape software to analyze and screen hub genes according to DC and $\mathrm{BC}$ parameter.

\section{GO and KEGG Pathway Enrichment Performance}

Gene Ontology (GO) analysis with the biological process (BP), cellular component (CC) and molecular function (MF) was carried out using the Database for Annotation, Visualization and Integrated Discovery (DAVID, version 6.7, https://david.ncifcrf.gov/)(26). Kyoto Encyclopedia of Genes and Genomes (KEGG) pathway enrichment was performed using ClueGO plugin in Cytoscape software, with species limited to "Homo sapiens" and P value $\leq 0.01$ (27). Then, the ClueGO results about KEGG pathway enrichment in TSV format were downloaded, and import into Cytoscape software to construct pathway-targets network.

\section{Results}

\section{Targets Screening of JTTZ and T2D with Obesity and Hyperlipidemia}

We obtained a total of 133 chemical ingredients of the eight herbal medicines in JTTZ from TCMSP, TCMID and TCM database@Taiwan. 110 compounds were finally selected after removing 23 compounds which did not link to any targets (Table 1), including 5 in Schisandrae Chinensis Fructus(WWZ), 55 in Radix Salviae(DS), 5 in Zingiberis Rhizoma(GJ), 14 in red yeast rice(HQ), 13 in Coptidis Rhizoma(HL), 4 in Momordica charantia(KG), 8 in Aloe vera(LH) and 12 in Anemarrhenae Rhizoma(ZM). We researched 8476 JTTZ-related targets from SWISS and PubChem. After removing duplication, 1235 potential targets of the ingredients were finally obtained, including 513 validated targets from PubChem and 722 predicted targets from SWISS. Sequentially, 368 potential therapeutic targets of T2D with obesity and hyperlipidemia. The data of 8476 JTTZ-related targets and 368 potential therapeutic targets were showed in Supplementary table 1. Finally, 166 candidate targets of JTTZ against T2D with obesity and hyperlipidemia were obtained and showed in Fig. 2.

\section{Compound-Targets Network Analysis}

Compound-targets network of JTTZ against T2D with obesity and hyperlipidemia was consisted of 276 nodes (110 compounds in JTTZ and 166 candidate targets) and 1423 edges which represented the interaction of compounds and targets (Fig. 2). We analyzed the network according to topological parameter including $\mathrm{DC}$ and $\mathrm{BC}$, which indicated the compounds and targets with more DC and BC is more important on the effect of JTTZ against T2D with obesity and hyperlipidemia. The results showed that the top five compounds included quercetin, arachidonic acid, kaempferol, luteolin and stigmasterol acted on 48 , $38,37,35$ and 33 candidate targets respectively. The OB represents the radio of the amount and speed absorbed into system circulation to achieve biological functions of oval drugs. The OB of quercetin, arachidonic acid, kaempferol, luteolin and stigmasterol is $46.43,45.57,41.88,36.16$ and 48.38 , respectively, which means they might be the crucial active compounds of JTTZ by reason of their considerable positioning in the network. 
Table 1

The final selected compounds in JTTZ for analysis.

\begin{tabular}{|c|c|c|c|c|c|c|c|c|c|}
\hline ID & Name & OB & $\mathrm{DL}$ & Source & ID & Name & OB & DL & Source \\
\hline MOL005317 & Deoxyharringtonine & 39.27 & 0.81 & WWZ & MOL007154 & tanshinone iia & 49.89 & 0.4 & DS \\
\hline MOL008956 & Angeloylgomisin 0 & 31.97 & 0.85 & WWZ & MOL006824 & a-amyrin & 39.51 & 0.76 & DS \\
\hline MOL008968 & Gomisin-A & 30.69 & 0.78 & WWZ & MOL007156 & 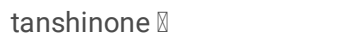 & 45.64 & 0.3 & DS \\
\hline MOL008974 & Gomisin G & 32.68 & 0.83 & WWZ & MOL000358 & beta-sitosterol & 36.91 & 0.75 & GJ \\
\hline MOL008992 & Wuweizisu C & 46.27 & 0.84 & WWZ & MOL002464 & 1-Monolinolein & 37.18 & 0.3 & GJ \\
\hline MOL000006 & luteolin & 36.16 & 0.25 & DS & MOL002514 & Sexangularetin & 62.86 & 0.3 & GJ \\
\hline MOL000569 & digallate & 61.85 & 0.26 & DS & MOL000359 & sitosterol & 36.91 & 0.75 & GJ LH \\
\hline MOL001659 & Poriferasterol & 43.83 & 0.76 & DS & MOL000702 & Guaijaverin & 29.65 & 0.7 & $\mathrm{HQ}$ \\
\hline MOL001942 & isoimperatorin & 45.46 & 0.23 & DS & MOL007155 & Daucosterol & 36.91 & 0.75 & $\mathrm{HQ}$ \\
\hline MOL002222 & sugiol & 36.11 & 0.28 & DS & MOL001965 & Dauricine & 23.65 & 0.37 & $\mathrm{HQ}$ \\
\hline MOL002776 & Baicalin & 40.12 & 0.75 & DS & MOL002320 & Gamma-Sitosterol & 36.91 & 0.75 & $\mathrm{HQ}$ \\
\hline MOL007143 & salvilenone I & 32.43 & 0.23 & DS & MOL005327 & ginsenoside Rd2_qt & 29.69 & 0.77 & $\mathrm{HQ}$ \\
\hline MOL007145 & salviolone & 31.72 & 0.24 & DS & MOL005376 & Panaxadiol & 33.09 & 0.79 & $\mathrm{HQ}$ \\
\hline MOL007149 & NSC 122421 & 34.49 & 0.28 & DS & MOL007475 & Ginsenoside f2 & 36.43 & 0.25 & $\mathrm{HQ}$ \\
\hline MOL007049 & 4-methylenemiltirone & 34.35 & 0.23 & DS & MOL009653 & Cycloeucalenol & 39.73 & 0.79 & $\mathrm{HQ}$ \\
\hline MOL002651 & Dehydrotanshinone II A & 43.76 & 0.40 & DS & MOL000098 & quercetin & 46.43 & 0.28 & $\begin{array}{l}\mathrm{HQ} H \mathrm{HL} \\
\mathrm{LH}\end{array}$ \\
\hline MOL007061 & Methylenetanshinquinone & 37.07 & 0.36 & DS & MOL012853 & Notoginsenoside R4-qt & 29.69 & 0.78 & $\mathrm{HQ}$ \\
\hline MOL007058 & formyltanshinone & 73.44 & 0.42 & DS & MOL007098 & deoxyneocryptotanshinone & 49.4 & 0.29 & DS \\
\hline MOL007094 & danshenspiroketallactone & 50.43 & 0.31 & DS & MOL000449 & Stigmasterol & 48.38 & 0.76 & $\begin{array}{l}\mathrm{HQ} \mathrm{KG} \\
\mathrm{ZM}\end{array}$ \\
\hline MOL007105 & epidanshenspiroketallactone & 68.27 & 0.31 & DS & MOL000622 & Magnograndiolide & 63.71 & 0.19 & $\mathrm{HL}$ \\
\hline MOL007077 & sclareol & 43.67 & 0.21 & DS & MOL000762 & Palmidin A & 35.36 & 0.65 & $\mathrm{HL}$ \\
\hline MOL007079 & tanshinaldehyde & 52.47 & 0.45 & DS & MOL000785 & palmatine & 64.6 & 0.65 & $\mathrm{HL}$ \\
\hline MOL007081 & Danshenol B & 57.95 & 0.56 & DS & MOL001454 & berberine & 36.86 & 0.78 & $\mathrm{HL}$ \\
\hline MOL007082 & Danshenol A & 56.97 & 0.52 & DS & MOL001458 & coptisine & 30.67 & 0.86 & $\mathrm{HL}$ \\
\hline MOL007085 & Salvilenone & 30.38 & 0.38 & DS & MOL002668 & Worenine & 45.83 & 0.87 & $\mathrm{HL}$ \\
\hline MOL007088 & cryptotanshinone & 52.34 & 0.4 & DS & MOL002894 & berberrubine & 35.74 & 0.73 & $\mathrm{HL}$ \\
\hline MOL007093 & dan-shexinkum d & 38.88 & 0.55 & DS & MOL002897 & epiberberine & 43.09 & 0.78 & $\mathrm{HL}$ \\
\hline MOL007100 & dihydrotanshinlactone & 38.68 & 0.32 & DS & MOL002903 & (R)-Canadine & 55.37 & 0.77 & $\mathrm{HL}$ \\
\hline MOL007101 & dihydrotanshinone区 & 45.04 & 0.36 & DS & MOL002904 & Berlambine & 36.68 & 0.82 & $\mathrm{HL}$ \\
\hline MOL002773 & Beta Carotene & 37.18 & 0.58 & HQ LH & MOL008647 & Moupinamide & 86.71 & 0.26 & $\mathrm{HL}$ \\
\hline MOL007107 & C09092 & 36.07 & 0.25 & DS & MOL013352 & Obacunone & 43.29 & 0.77 & $\mathrm{HL}$ \\
\hline MOL007108 & isocryptotanshi-none & 54.98 & 0.39 & DS & MOL008569 & $\beta$-sitosterol- $\beta$-d-glucoside & 32.41 & 0.71 & KG \\
\hline MOL007064 & przewalskin b & 110.3 & 0.44 & DS & MOL010267 & lycopene & 32.57 & 0.51 & KG \\
\hline MOL007111 & Isotanshinone II & 49.92 & 0.4 & DS & MOL012374 & momordic acid & 36.36 & 0.75 & KG \\
\hline MOL007115 & manool & 45.04 & 0.2 & DS & MOL000471 & aloe-emodin & 83.38 & 0.24 & $\mathrm{LH}$ \\
\hline MOL007118 & microstegiol & 39.61 & 0.28 & DS & MOL000953 & CLR & 37.87 & 0.68 & LH \\
\hline MOL007119 & miltionone $\nabla$ & 49.68 & 0.32 & DS & MOL001439 & arachidonic acid & 45.57 & 0.2 & $\mathrm{LH}$ \\
\hline MOL007120 & miltionone $\mathbb{Q}$ & 71.03 & 0.44 & DS & MOL005051 & Aloeresin C & 34.99 & 0.5 & $\mathrm{LH}$ \\
\hline MOL007121 & miltipolone & 36.56 & 0.37 & DS & MOL000422 & kaempferol & 41.88 & 0.24 & ZM \\
\hline MOL007122 & Miltirone & 38.76 & 0.25 & DS & MOL000546 & diosgenin & 80.88 & 0.81 & ZM \\
\hline
\end{tabular}




\begin{tabular}{|c|c|c|c|c|c|c|c|c|c|}
\hline ID & Name & $\mathrm{OB}$ & DL & Source & ID & Name & OB & $\mathrm{DL}$ & Source \\
\hline MOL007123 & miltirone $\mathbb{Z}$ & 44.95 & 0.24 & DS & MOL000631 & coumaroyltyramine & 112.9 & 0.2 & ZM \\
\hline MOL007124 & neocryptotanshinone ii & 39.46 & 0.23 & DS & MOL001677 & asperglaucide & 58.02 & 0.52 & ZM \\
\hline MOL007125 & neocryptotanshinone & 52.49 & 0.32 & DS & MOL003773 & Mangiferolic acid & 36.16 & 0.84 & ZM \\
\hline MOL007130 & prolithospermic acid & 64.37 & 0.31 & DS & MOL004373 & Anhydroicaritin & 45.41 & 0.44 & ZM \\
\hline MOL007141 & salvianolic acid g & 45.56 & 0.61 & DS & MOL004497 & Hippeastrine & 51.65 & 0.62 & ZM \\
\hline MOL007142 & salvianolic acid $\mathrm{j}$ & 43.38 & 0.72 & DS & MOL004489 & Anemarsaponin F_qt & 60.06 & 0.79 & ZM \\
\hline MOL004540 & Anemarsaponin C_qt & 35.5 & 0.87 & ZM & MOL005043 & campest-5-en-3beta-ol & 37.58 & 0.71 & LH \\
\hline MOL004542 & Anemarsaponin E_qt & 30.67 & 0.86 & ZM & MOL006730 & Notoginsenoside Fa_qt & 29.69 & 0.77 & $\mathrm{HQ}$ \\
\hline MOL001771 & poriferast-5-en-3beta-ol & 36.91 & 0.75 & DS & MOL007041 & $\begin{array}{l}\text { 2-isopropyl-8- } \\
\text { methylphenanthrene-3,4-dione }\end{array}$ & 40.86 & 0.23 & DS \\
\hline MOL001601 & 1,2,5,6-tetrahydrotanshinone & 38.75 & 0.36 & DS & MOL011455 & 20-Hexadecanoylingenol & 32.7 & 0.65 & HQ \\
\hline MOL007051 & $\begin{array}{l}\text { 6-o-syringyl-8-o-acetyl- } \\
\text { shanzhiside methyl ester }\end{array}$ & 46.69 & 0.71 & DS & MOL007127 & $\begin{array}{l}\text { 1-methyl-8,9-dihydro-7H- } \\
\text { naphtho[5,6-g]benzofuran- } \\
6,10,11 \text {-trione }\end{array}$ & 34.72 & 0.37 & DS \\
\hline MOL002501 & $\begin{array}{l}\text { [(1S)-3-[(E)-but-2-enyl]-2- } \\
\text { methyl-4-oxo-1-cyclopent-2- } \\
\text { enyl] (1R,3R)-3-[(E)-3- } \\
\text { methoxy-2-methyl-3-oxoprop- } \\
\text { 1-enyl]-2,2- } \\
\text { dimethylcyclopropane-1- } \\
\text { carboxylate }\end{array}$ & 62.52 & 0.31 & GJ & MOL007155 & $\begin{array}{l}\text { (6S)-6-(hydroxymethyl)-1,6- } \\
\text { dimethyl-8,9-dihydro-7H-- } \\
\text { naphtho[8,7-g]benzofuran- } \\
\text { 10,11-dione }\end{array}$ & 65.26 & 0.45 & DS \\
\hline MOL007132 & $\begin{array}{l}(2 \mathrm{R})-3-(3,4- \\
\text { dihydroxyphenyl)-2-[(Z)-3- } \\
\text { (3,4-- } \\
\text { dihydroxyphenyl)acryloyl]oxy- } \\
\text { propionic acid }\end{array}$ & 109.4 & 0.35 & DS & MOL007048 & $\begin{array}{l}\text { (E)-3-[2-(3,4-dihydroxyphenyl)-7- } \\
\text { hydroxy-benzofuran-4-yl]acrylic } \\
\text { acid }\end{array}$ & 48.24 & 0.31 & DS \\
\hline MOL007140 & $\begin{array}{l}\text { (Z)-3-[2-[(E)-2-(3,4- } \\
\text { dihydroxyphenyl)vinyl]-3,4- } \\
\text { dihydroxy-phenyl]acrylic acid }\end{array}$ & 88.54 & 0.26 & DS & MOL007036 & $\begin{array}{l}\text { 5,6-dihydroxy-7-isopropyl-1,1- } \\
\text { dimethyl-2,3- } \\
\text { dihydrophenanthren-4-one }\end{array}$ & 33.77 & 0.29 & DS \\
\hline MOL007050 & $\begin{array}{l}\text { 2-(4-hydroxy-3- } \\
\text { methoxyphenyl)-5-(3- } \\
\text { hydroxypropyl)-7-methoxy-3- } \\
\text { benzofurancarboxaldehyde }\end{array}$ & 62.78 & 0.4 & DS & MOL000483 & $\begin{array}{l}\text { (Z)-3-(4-hydroxy-3-methoxy- } \\
\text { phenyl)-N-[2-(4- } \\
\text { hydroxyphenyl)ethyl]acrylamide }\end{array}$ & 118.35 & 0.26 & ZM \\
\hline
\end{tabular}

\section{PPI Networks Analysis}

The PPI network of JTTZ against T2D with obesity and hyperlipidemia-related candidate targets contained 164 nodes and 1735 edges, which illustrated 164 interacting proteins and 1735 interactions (Fig. 3A). The protein tends to form macromolecular complexes by interaction to accomplish biological functions, so the PPI network play a vital role in mechanism analysis of biological processes. In order to select the more important interactive proteins, we set indexes of confidence score $\geq 0.9$ and DC $\geq 8.294$ (the average value of DC) to re-constructed the PPI network containing 59 nodes and 375 edges (Fig. $3 B$ ). The targets were further screened and 19 targets with $B C \geq 0.034$ (the median values of $B C$ ), which were involved in various pathogenic processes of $T 2 D$ with obesity and hyperlipidemia (Fig. 3C).

\section{GO and KEGG Pathway Enrichment Analysis}

GO of 166 candidate targets was analyzed based on BP, CC and MF, and the top 20 was screened out, respectively (Fig. 4). The results indicated that BP of these targets protein related to inflammatory response, steroid and bile acid metabolism, cellular proliferation and apoptotic, insulin secretion, vasoconstriction, response to hypoxia, and oxidation-reduction process, such as inflammatory response referring to G-protein coupled receptor signaling pathway and response to lipopolysaccharide. CC is mainly distributed in integral component of plasm, integral and partial component of membrane, nuclear chromatin, endoplasmic reticulum body and membrane, neuronal cell body, projection and dendrite, receptor complex, endocytic vesicle and extracellular exosome. And MF contains molecular activities like G-protein coupled serotonin receptor, steroid hormone receptor, and molecular bind, such as steroid, drug, enzyme, ligand-activated sequence-specific DNA, coupled to transmembrane movement of substances, coupled via Gs.

KEGG pathway analysis was performed to show that these targets were mainly enriched in 13 pathway by ClueGO plugin (Fig. 5), including AGE-RAGE signaling pathway in diabetic complications, AMPK signaling pathway, Adipocytokine signaling pathway, Bile secretion, Calcium signaling pathway, Cocaine addiction, Neuroactive ligand-receptor interaction, Pathways in cancer, Serotonergic synapse, Steroid hormone biosynthesis, Thyroid hormone signaling pathway, cAMP signaling pathway and cGMP-PKG signaling pathway, and these pathways were linked by related genes interaction. The data of KEGG pathway enrichment analysis were shown in Supplementary table 2.

\section{Identified Candidate Targets by Analysis of Pathway-Targets Network}


To further explore the mechanism of JTTZ in treatment of T2D with obesity and hyperlipidemia. The pathway-targets network is constructed based on the significantly enriched pathways and genes regulating these pathways (Fig. 6). The network contains 148 nodes (13 in pathways and 135 in related-genes) and 337 edges. The topological analysis results showed that adipocytokine signaling pathway has the most maximum BC with 48 directed edges and the main 10 targets included AKT2, PRKCB, RELA, NFKB1, GSK3B, BRAF, EDNRA, PTGS2, SLC2A1, PDGFRB and HDAC2.

\section{Discussion}

In our previous studies, we discovered that JTTZ could potentially be developed as an alternative medicine because it appeared to alleviate symptoms of T2D with obesity and hyperlipidemia. However, the molecule mechanism and action targets is still unclear. Therefore, we explored possible mechanism of JTTZ against T2D with obesity and hyperlipidemia by the TCM network pharmacology.

The JTTZ formula consists of eight herbs to be used for stomach heat syndrome in clinical application. In the present study, a compound-targets network of JTTZ was constructed containing 110 compounds and 166 targets. The results showed that the most compounds acted on multiple targets, such as quercetin, arachidonic acid, kaempferol, luteolin and stigmasterol effecting 48, 38, 37, 35 and 33 candidate targets respectively, which means they might be the crucial active compounds of JTTZ by reason of their considerable positioning in the network. Quercetin is a polyphenol, and have significant effects on anti-inflammation, anti-obesity and anti-diabetes through enhancing antioxidative activities, regulating MAPK and NF-KB/JNK pathway, reducing the proinflammation enzymes activity and cytokine levels(28). Arachidonic acid is a strong inducer of insulin secretion and its metabolism is involved in free fatty acids and hyperglycemia mediated inflammation base on lipid and immune pathway(29). Kaempferol is a kind of natural flavonoids and has been exerted extensive biological activities, for example, anti-oxidation, anti-inflammation, antibacterial and antitumor activities, moreover, it also show a notable inhibition activity on a-glucosidase(30). Luteolin also is a represent flavonoid and it could alleviate fibrosis, hypertrophy and apoptosis, and inhibit inflammation and oxidative stress through inhibition of NF-KB and induction of the Nrf2 pathway(31). Stigmasterol is a minor phytosterol and showed reducing plasm cholesterol by decreasing cholesterol absorption, suppressing hepatic bile acid and cholesterol synthesis, and stimulating transintestinal cholesterol excretion mediated by liver $X$ receptor activation(32). Although the biological functions of single herbal compounds is different, due to overlapping targets in network, the multiple compounds of JTTZ would act on same targets and pathways to achieve synergistic effects.

The main pathological mechanism of T2D with obesity and hyperlipidemia refers to variety organs, such as pancreas, liver, adipose tissue, skeletal muscle, kidney, brain and small intestine, and is characterized by decreased insulin sensitivity, common insulin resistance, pancreatic $\beta$-cell failure, adipose tissue dysfunction, sterol and bile acids metabolic disorders and chronic systematic inflammation(2, 5). By the PPI, GO and KEGG pathway enrichment analysis, biological process of JTTZ related to inflammatory response, steroid and bile acid metabolism, cellular proliferation and apoptotic, insulin secretion, response to hypoxia, vasoconstriction, and oxidation-reduction process, and major pathways contained adipocytokine signaling pathway, AMPK signaling pathway, CAMP signaling pathway and CGMP-PKG signaling pathway, NF-KB and MAPK signaling pathway, we summarized several potential action mechanisms and therapeutic targets, including adipose tissue dysfunction, inflammation response, and insulin resistance (Fig. 7).

\section{Adipose tissue dysfunction}

Adipose tissue, which main included white adipose tissue (WAT) and brown adipose tissue (BAT), regulates multiple physiological processes and its dysfunction is associated with imbalance of metabolic homeostasis, insulin resistance, adiposity and T2D through dysregulated adipokine secretion, disrupted mitochondrial function, inflammation, fibrosis, and hypoxia $(33,34)$. On the one hand, WAT play an important role in energy storage in the form of free fatty acids (FFA), which would maintain lipid metabolic homeostasis based on triacylglycerol (TAG) hydrolysis by lipoprotein lipase (LPL) activity to move non-esterified fatty acids (NEFA) into adipocytes and triacylglycerols (TAGs) lipolysis via cAMP activates protein kinases A (PKA) and cGMP activates protein kinases G (PKG) leading to phosphorylation of perilipin and hormone-sensitive lipase $(\mathrm{HSL})(35,36)$. In addition, the activated AMPK exerts the antilipolytic effects on adipocytes by regulating HSL controlled by PPARY $(37,38)$. PPARY is key regulator of adipocyte differentiation and glucose homeostasis, whose agonist commonly used to treat diabetes(39). And BAT are important in thermogenesis via generating ATP within a large number of mitochondria for energy production(35). GO and KEGG pathway enrichment analysis also showed JTTZ could ameliorate T2D patients with obesity and hyperlipidemia via AMPK signaling pathway, cAMP signaling pathway and cGMP-PKG signaling pathway. On the other hand, adipose tissue is a remarkably complex endocrine organ to secret a number of adipocyte-derived factors called as adipokines, such as leptin and adiponectin derived from WAT, insulin-like growth factor 1 (IGF-1) derived from BAT, and a variety of proinflammatory cytokines like tumor necrosis factor (TNFa), interleukin-1 $\beta$ (IL-1 $\beta$ ) and interleukin$6(\mathrm{IL}-6)(34)$. Our present study showed adipocytokine signaling pathway had the most maximum BC and occupied an important position in pathway-targets network. The past studies revealed that both leptin and adiponectin could promote increased insulin sensitivity and glucose tolerance via activities of AMPK and exerting anti-inflammatory effects through IGF-1 and the inducement of M2 macrophages, respectively(40-42). Moreover, expanded adipose tissue synthesizes and secretes cytokines including TNFa, IL-1 $\beta$, IL-6, which cause adipose tissue inflammation that may be a driving factor in the development of systemic IR and T2D in obesity $(34,43)$. Therefore, the regulation of dysfunctional adipose tissue may be potential therapeutic strategies target for JTTZ formula against T2D with obesity and hyperlipidemia.

Figure 7: 1.JTTZ could improve glucose and lipid homeostasis by inhibiting cAMP-PKA signaling pathway and cGMP-PKG signaling pathway. 2.JTTZ could regulate secretion of adipose tissue, which exert activation to increase adipokines (leptin and adiponectin) and inactivation to decrease proinflammatory cytokines (TNFa, IL-1 $\beta$, IL-6) via shifting M1 macrophages to M2 macrophages for anti-inflammation. 3. JTTZ could suppress inflammation through inhibiting NF-KB and MAPK signaling pathways. 4. JTTZ achieve effect on amelioration of insulin resistance by acting on AKT2 and GSK3 therapeutic targets. JTTZ, Jiangtang Tiaozhi; cAMP, cyclic adenosine monophosphate; PKA, protein kinases A; cGMP, cyclic guanosine monophosphate; PKG, protein kinases G; HSL, hormone-sensitive lipase; TAGs, triacylglycerols; NEFA, non-esterified fatty acids; LPS, lipopolysaccharide; LBP, lipopolysaccharide binding protein; CD14, differentiation; TLR4, toll-like receptors; MyD88, myeloid differentiation primary response gene; TNFa, tumor necrosis factor; IL-1 $\beta$, interleukin-

Page 6/15 
1ß; IL-6, interleukin-6; TNFR, TNF receptor; ILR, interleukin receptor; TRAF, TNF receptor-associated factor; IRAK, interleukin receptor-associated kinase; IKKs, Ikappa-B kinases; IKB, I-kappa-B; NFKB, nuclear factor- KB; MAPK, mitogen-activated protein kinase; JNK, jun N-terminal kinase; IRS, insulin receptor substrates; PI3K, phosphatidylinositol-3-kinase; AKT2, serine/threonine protein kinase B; GSK3, glycogen synthase kinase-3; GS, glycogen synthesis.

\section{Systematic inflammation}

The results of GO and KEGG pathway analysis showed inflammation referred to response to lipopolysaccharide and NF-KB and MAPK signaling pathway. Obesity-induced diabetes is accompanied with a chronic low grade inflammation, which may in part be mediated by endotoxins (especially lipopolysaccharide, LPS) derived from gram-negative bacterial species $(44,45)$. LPS toxicity is triggered by lipid A component via recognition of Toll-like receptors (TLR), but LPS has a short half-life, so the complex of LPS-lipopolysaccharide binding protein (LBP) to cluster of differentiation (CD14) leads to inflammatory responses via activation of TLR4 $(45,46)$. The activation of TLR4 interacting with LPS could trigger various signaling transduction, mainly including NF-KB and MAPK(47). NF-KB is a pleiotropic transcription factor present in almost all cell types and is the endpoint of a series of signal transduction events, which is a homo- or heterodimeric complex formed by the Rel-like domain-containing proteins RELA/p65, RELB, NFKB1/p105, NFKB1/p50, et al $(48,49)$. Our pathway-targets network indicates that RELA and NFKB1 may be therapeutic targets of JTTZ formula against T2D with obesity and hyperlipidemia. NF-KB is in an inactive state because of its combination with l-kappa-B (IKB). The interaction with LPS and TLR4 leads to the phosphorylation of I-kappa-B kinases (IKKs) complex via myeloid differentiation primary response gene (MyD88), IL-1 receptor-associated kinase (IRAK), TNF receptor-associated factor (TRAF), NF-KB inducing kinase (NIK), which causes that IKB is phosphorylated to degrade NF-KB complex for translocation to the nucleus and subsequent expression of pro-inflammatory genes $(47,49)$. MAPK represent a family of kinases for phosphorylation of serine and threonine, whose signaling pathway includes the extracellular signal-regulated kinases such as JNK, p38 MAPK and extracellular signal-related kinase(50). The activation of TLR4 stimulates JNK and p38 MAPK signaling pathway(51). JNK exerts direct inhibitory phosphorylation of insulin receptor substrates (IRS) 1 and 2 leading to decrease recruitment of the phosphatidylinositol-3-kinase (PI3K)- serine/threonine protein kinase B (AKT2) signaling pathway in response to insulin, otherwise, JNK play a major role in obesity-induced macrophage activation leading to increase levels of M1 macrophages to induce inflammatory cytokines driving insulin resistance $(50,52)$. p38 MAPK activation reduces the expression of IRS1 and IRS2 contributing to insulin resistance, and accelerate biosynthesis of inflammatory cytokines such as TNFa, IL-1 $\beta$ and IL-6 in LPS-stimulated human monocytes $(53,54)$.

\section{Insulin resistance}

The pathological state of insulin resistance is associated with obesity-induced T2D, moreover, our the analysis of network pharmacology revealed that JTTZ formula may be act on insulin signaling pathway especially AKT2 and glycogen synthase kinase-3 (GSK3) gene targets to treat T2D with obesity and hyperlipidemia(55). The classic insulin signaling starts when it binds to IRS1 and IRS2, which the phosphorylation of these proteins enable trigger $\mathrm{PI3K} / \mathrm{AKT} 2$ pathway to regulate insulin metabolic actions $(47,55)$. AKT2 is responsible of the regulation of glucose uptake by mediating insulin-induced translocation of the SLC2A4/GLUT4 glucose transporter to the cell surface, and is contributed to the storage of glucose in the form of glycogen by phosphorylating GSK3 $(56,57)$. The phosphorylating of GSK3 is inactive action to eliminate the activation of glycogen synthesis (GS) and glucose uptake(58). GSK3 is a multi-functional serine/threonine kinase which is increased expression in the skeletal muscle of patients withT2D, and GSK3 inhibitors have been regarded as an anti-diabetic therapeutic target $(59,60)$.

\section{Conclusion}

Our study systematically explored the potential action mechanism and therapeutic targets of JTTZ formula against obesity and hyperlipidemia used network pharmacologic method. Although JTTZ formula have variety components involved in multiple targets, different compounds could act on same targets via multiple biological process and signaling pathway. The results indicate that JTTZ formula could achieve effects on anti-diabetes, anti-obesity and anti-hyperlipidemia through regulation and improvement of adipose tissue dysfunction, systematic inflammation and insulin resistance, which may be mediated by multiple targets, such as RELA, NFKB, AKT2, GSK3, et al. However, there is still some limitation in this study due to network research and machine algorithms, therefore, it is necessary to further validate mechanisms of JTTZ formula by experiments.

\section{Abbreviations}

JTTZ, Jiangtang Tiaozhi; cAMP, cyclic adenosine monophosphate; PKA, protein kinases A; cGMP, cyclic guanosine monophosphate; PKG, protein kinases G; HSL, hormone-sensitive lipase; TAGs, triacylglycerols; NEFA, non-esterified fatty acids; LPS, lipopolysaccharide; LBP, lipopolysaccharide binding protein; CD14, differentiation; TLR4, toll-like receptors; MyD88, myeloid differentiation primary response gene; TNFa, tumor necrosis factor; IL-1 $\beta$, interleukin-1 $\beta$; IL-6, interleukin-6; TNFR, TNF receptor; ILR, interleukin receptor; TRAF, TNF receptor-associated factor; IRAK, interleukin receptor-associated kinase; IKKs, I-kappa-B kinases; IKB, I-kappa-B; NFKB, nuclear factor- KB; MAPK, mitogen-activated protein kinase; JNK, jun N-terminal kinase; IRS, insulin receptor substrates; PI3K, phosphatidylinositol-3-kinase; AKT2, serine/threonine protein kinase B; GSK3, glycogen synthase kinase-3; GS, glycogen synthesis.

\section{Declarations}

\section{Ethics approval and consent to participate}

Not applicable

\section{Consent to publish}


Not applicable

\section{Availability of data and materials}

The data used to support the results of this study can be obtained from the corresponding author.

\section{Competing interests}

The authors declare that the research was conducted in the absence of any commercial or financial relationships that could be construed as a potential conflict of interest.

\section{Funding}

This work was supported by the National Public Welfare Industry Special (201507001-11) and National Natural Science Foundation of China (No. 81904187).

\section{Authors' Contributions}

FL and XT initiated the study. HZ performed the data and literature search, drafted the original manuscript, and drew the figures. XA and DJ helped for introduction and discussion. JT, SZ, XW, YY and WL contributed to revisions. All authors approved the final version of the manuscript.

\section{Acknowledgements}

Not applicable

\section{References}

1. Franks PW, McCarthy MI. Exposing the exposures responsible for type 2 diabetes and obesity. Science. 2016;354(6308):69-73.

2. Roden M, Shulman GI. The integrative biology of type 2 diabetes. Nature. 2019;576(7785):51-60.

3. Findeisen M, Allen TL, Henstridge DC, Kammoun H, Brandon AE, Baggio LL, et al. Treatment of type 2 diabetes with the designer cytokine IC7Fc. Nature. 2019;574(7776):63-8.

4. Sinclair A, Saeedi P, Kaundal A, Karuranga S, Malanda B, Williams R. Diabetes and global ageing among 65-99-year-old adults: Findings from the International Diabetes Federation Diabetes Atlas, 9(th) edition. Diabetes Res Clin Pract. 2020:108078.

5. Chatterjee S, Khunti K, Davies MJ. Type 2 diabetes. Lancet. 2017;389(10085):2239-51.

6. Kahn SE, Hull RL, Utzschneider KM. Mechanisms linking obesity to insulin resistance and type 2 diabetes. Nature. 2006;444(7121):840-6.

7. Foretz M, Guigas B, Viollet B. Understanding the glucoregulatory mechanisms of metformin in type 2 diabetes mellitus. Nat Rev Endocrinol. 2019;15(10):569-89.

8. Leitner DR, Fruhbeck G, Yumuk V, Schindler K, Micic D, Woodward E, et al. Obesity and Type 2 Diabetes: Two Diseases with a Need for Combined Treatment Strategies - EASO Can Lead the Way. Obes Facts. 2017;10(5):483-92.

9. Yu X, Xu L, Zhou Q, Wu S, Tian J, Piao C, et al. The Efficacy and Safety of the Chinese Herbal Formula, JTTZ, for the Treatment of Type 2 Diabetes with Obesity and Hyperlipidemia: A Multicenter Randomized, Positive-Controlled, Open-Label Clinical Trial. Int J Endocrinol. 2018;2018:9519231.

10. Hopkins AL. Network pharmacology: the next paradigm in drug discovery. Nat Chem Biol. 2008;4(11):682-90.

11. Li S, Zhang B. Traditional Chinese medicine network pharmacology: theory, methodology and application. Chin J Nat Med. 2013;11(2):110-20.

12. Li S. Exploring traditional chinese medicine by a novel therapeutic concept of network target. Chin J Integr Med. 2016;22(9):647-52.

13. Wang R, Lin J. Analysis of the Mechanism of Zhichuanling Oral Liquid in Treating Bronchial Asthma Based on Network Pharmacology. Evid Based Complement Alternat Med. 2020;2020:1875980.

14. Jiang Y, Liu N, Zhu S, Hu X, Chang D, Liu J. Elucidation of the Mechanisms and Molecular Targets of Yiqi Shexue Formula for Treatment of Primary Immune Thrombocytopenia Based on Network Pharmacology. Front Pharmacol. 2019;10:1136.

15. Liang X, Li H, Li S. A novel network pharmacology approach to analyse traditional herbal formulae: the Liu-Wei-Di-Huang pill as a case study. Mol Biosyst. 2014;10(5):1014-22.

16. Ru J, Li P, Wang J, Zhou W, Li B, Huang C, et al. TCMSP: a database of systems pharmacology for drug discovery from herbal medicines. J Cheminform. 2014;6:13.

17. Xue R, Fang Z, Zhang M, Yi Z, Wen C, Shi T. TCMID: Traditional Chinese Medicine integrative database for herb molecular mechanism analysis. Nucleic Acids Res. 2013;41(Database issue):D1089-95.

18. Chen CY. TCM Database@Taiwan: the world's largest traditional Chinese medicine database for drug screening in silico. PLoS One. 2011;6(1):e15939. 
19. Gfeller D, Grosdidier A, Wirth M, Daina A, Michielin O, Zoete V. SwissTargetPrediction: a web server for target prediction of bioactive small molecules. Nucleic Acids Res. 2014;42(Web Server issue):W32-8.

20. Consortium TU. UniProt: a worldwide hub of protein knowledge. Nucleic Acids Res. 2019;47(D1):D506-d15.

21. Wang Y, Zhang S, Li F, Zhou Y, Zhang Y, Wang Z, et al. Therapeutic target database 2020: enriched resource for facilitating research and early development of targeted therapeutics. Nucleic Acids Res. 2020;48(D1):D1031-d41.

22. Wishart DS, Feunang YD, Guo AC, Lo EJ, Marcu A, Grant JR, et al. DrugBank 5.0: a major update to the DrugBank database for 2018. Nucleic Acids Res. 2018;46(D1):D1074-d82.

23. Pinero J, Bravo A, Queralt-Rosinach N, Gutierrez-Sacristan A, Deu-Pons J, Centeno E, et al. DisGeNET: a comprehensive platform integrating information on human disease-associated genes and variants. Nucleic Acids Res. 2017;45(D1):D833-d9.

24. Su G, Morris JH, Demchak B, Bader GD. Biological network exploration with Cytoscape 3. Curr Protoc Bioinformatics. 2014;47:8.13.1-24.

25. Szklarczyk D, Morris JH, Cook H, Kuhn M, Wyder S, Simonovic M, et al. The STRING database in 2017: quality-controlled protein-protein association networks, made broadly accessible. Nucleic Acids Res. 2017;45(D1):D362-d8.

26. Jiao X, Sherman BT, Huang da W, Stephens R, Baseler MW, Lane HC, et al. DAVID-WS: a stateful web service to facilitate gene/protein list analysis. Bioinformatics. 2012;28(13):1805-6.

27. Bindea G, Mlecnik B, Hackl H, Charoentong P, Tosolini M, Kirilovsky A, et al. ClueGO: a Cytoscape plug-in to decipher functionally grouped gene ontology and pathway annotation networks. Bioinformatics. 2009;25(8):1091-3.

28. Chen S, Jiang H, Wu X, Fang J. Therapeutic Effects of Quercetin on Inflammation, Obesity, and Type 2 Diabetes. Mediators Inflamm. 2016;2016:9340637.

29. Sonnweber T, Pizzini A, Nairz M, Weiss G, Tancevski I. Arachidonic Acid Metabolites in Cardiovascular and Metabolic Diseases. Int J Mol Sci. 2018;19(11).

30. Peng X, Zhang G, Liao Y, Gong D. Inhibitory kinetics and mechanism of kaempferol on alpha-glucosidase. Food Chem. 2016;190:207-15.

31. Li L, Luo W, Qian Y, Zhu W, Qian J, Li J, et al. Luteolin protects against diabetic cardiomyopathy by inhibiting NF-kappaB-mediated inflammation and activating the Nrf2-mediated antioxidant responses. Phytomedicine. 2019;59:152774.

32. Lifsey HC, Kaur R, Thompson BH, Bennett L, Temel RE, Graf GA. Stigmasterol stimulates transintestinal cholesterol excretion independent of liver $X$ receptor activation in the small intestine. J Nutr Biochem. 2020;76:108263.

33. Kusminski CM, Bickel PE, Scherer PE. Targeting adipose tissue in the treatment of obesity-associated diabetes. Nat Rev Drug Discov. 2016;15(9):639-60.

34. Saxton SN, Clark BJ, Withers SB, Eringa EC, Heagerty AM. Mechanistic Links Between Obesity, Diabetes, and Blood Pressure: Role of Perivascular Adipose Tissue. Physiol Rev. 2019;99(4):1701-63.

35. Lafontan M, Langin D. Lipolysis and lipid mobilization in human adipose tissue. Prog Lipid Res. 2009;48(5):275-97.

36. Zhang HH, Halbleib M, Ahmad F, Manganiello VC, Greenberg AS. Tumor necrosis factor-alpha stimulates lipolysis in differentiated human adipocytes through activation of extracellular signal-related kinase and elevation of intracellular cAMP. Diabetes. 2002;51(10):2929-35.

37. Zhong J, Gong W, Lu L, Chen J, Lu Z, Li H, et al. Irbesartan ameliorates hyperlipidemia and liver steatosis in type 2 diabetic db/db mice via stimulating PPAR-gamma, AMPK/Akt/mTOR signaling and autophagy. Int Immunopharmacol. 2017;42:176-84.

38. Song Y, Li X, Liu Y, Hu Y, Yang R. Arctigenin improves lipid metabolism by regulating AMP-activated protein kinase and downstream signaling pathways. J Cell Biochem. 2019;120(8):13275-88.

39. Wong WT, Tian XY, Xu A, Yu J, Lau CW, Hoo RL, et al. Adiponectin is required for PPARgamma-mediated improvement of endothelial function in diabetic mice. Cell Metab. 2011;14(1):104-15.

40. de Boer MP, Meijer RI, Richter EA, van Nieuw Amerongen GP, Sipkema P, van Poelgeest EM, et al. Globular adiponectin controls insulin-mediated vasoreactivity in muscle through AMPKalpha2. Vascul Pharmacol. 2016;78:24-35.

41. Hui X, Gu P, Zhang J, Nie T, Pan Y, Wu D, et al. Adiponectin Enhances Cold-Induced Browning of Subcutaneous Adipose Tissue via Promoting M2 Macrophage Proliferation. Cell Metab. 2015;22(2):279-90.

42. Ropelle ER, Fernandes MF, Flores MB, Ueno M, Rocco S, Marin R, et al. Central exercise action increases the AMPK and mTOR response to leptin. PLoS One. 2008;3(12):e3856.

43. Burhans MS, Hagman DK, Kuzma JN, Schmidt KA, Kratz M. Contribution of Adipose Tissue Inflammation to the Development of Type 2 Diabetes Mellitus. Compr Physiol. 2018;9(1):1-58.

44. Pussinen PJ, Havulinna AS, Lehto M, Sundvall J, Salomaa V. Endotoxemia is associated with an increased risk of incident diabetes. Diabetes Care. 2011;34(2):392-7.

45. Gomes JMG, Costa JA, Alfenas RCG. Metabolic endotoxemia and diabetes mellitus: A systematic review. Metabolism. 2017;68:133-44.

46. Tuomi K, Logomarsino JV. Bacterial Lipopolysaccharide, Lipopolysaccharide-Binding Protein, and Other Inflammatory Markers in Obesity and After Bariatric Surgery. Metab Syndr Relat Disord. 2016;14(6):279-88.

47. Boroni Moreira AP, de Cassia Goncalves Alfenas R. The influence of endotoxemia on the molecular mechanisms of insulin resistance. Nutr Hosp. 2012;27(2):382-90.

48. Dominguez-Acosta O, Vega L, Estrada-Muniz E, Rodriguez MS, Gonzalez FJ, Elizondo G. Activation of aryl hydrocarbon receptor regulates the LPS/IFNgamma-induced inflammatory response by inducing ubiquitin-proteosomal and lysosomal degradation of RelA/p65. Biochem Pharmacol. 2018;155:141-9.

Page 9/15 
49. Baker RG, Hayden MS, Ghosh S. NF-kappaB, inflammation, and metabolic disease. Cell Metab. 2011;13(1):11-22.

50. Hirosumi J, Tuncman G, Chang L, Gorgun CZ, Uysal KT, Maeda K, et al. A central role for JNK in obesity and insulin resistance. Nature. 2002;420(6913):333-6.

51. Chistyakov DV, Azbukina NV, Astakhova AA, Polozhintsev Al, Sergeeva MG, Reiser G. Toll-like receptors control p38 and JNK MAPK signaling pathways in rat astrocytes differently, when cultured in normal or high glucose concentrations. Neurochem Int. 2019;131:104513.

52. Solinas G, Becattini B. JNK at the crossroad of obesity, insulin resistance, and cell stress response. Mol Metab. 2017;6(2):174-84.

53. Kumar S, Boehm J, Lee JC. p38 MAP kinases: key signalling molecules as therapeutic targets for inflammatory diseases. Nat Rev Drug Discov. 2003;2(9):717-26.

54. Wang S, Ding L, Ji H, Xu Z, Liu Q, Zheng Y. The Role of p38 MAPK in the Development of Diabetic Cardiomyopathy. Int J Mol Sci. 2016;17(7).

55. Petersen MC, Shulman GI. Mechanisms of Insulin Action and Insulin Resistance. Physiol Rev. 2018;98(4):2133-223.

56. Ng Y, Ramm G, Lopez JA, James DE. Rapid activation of Akt2 is sufficient to stimulate GLUT4 translocation in 3T3-L1 adipocytes. Cell Metab. 2008;7(4):348-56.

57. Hussain K, Challis B, Rocha N, Payne F, Minic M, Thompson A, et al. An activating mutation of AKT2 and human hypoglycemia. Science. 2011;334(6055):474.

58. Beurel E, Grieco SF, Jope RS. Glycogen synthase kinase-3 (GSK3): regulation, actions, and diseases. Pharmacol Ther. 2015;148:114-31.

59. Rayasam GV, Tulasi VK, Sodhi R, Davis JA, Ray A. Glycogen synthase kinase 3: more than a namesake. Br J Pharmacol. 2009;156(6):885-98.

60. Nikoulina SE, Ciaraldi TP, Mudaliar S, Mohideen P, Carter L, Henry RR. Potential role of glycogen synthase kinase-3 in skeletal muscle insulin resistance of type 2 diabetes. Diabetes. 2000;49(2):263-71.

\section{Figures}

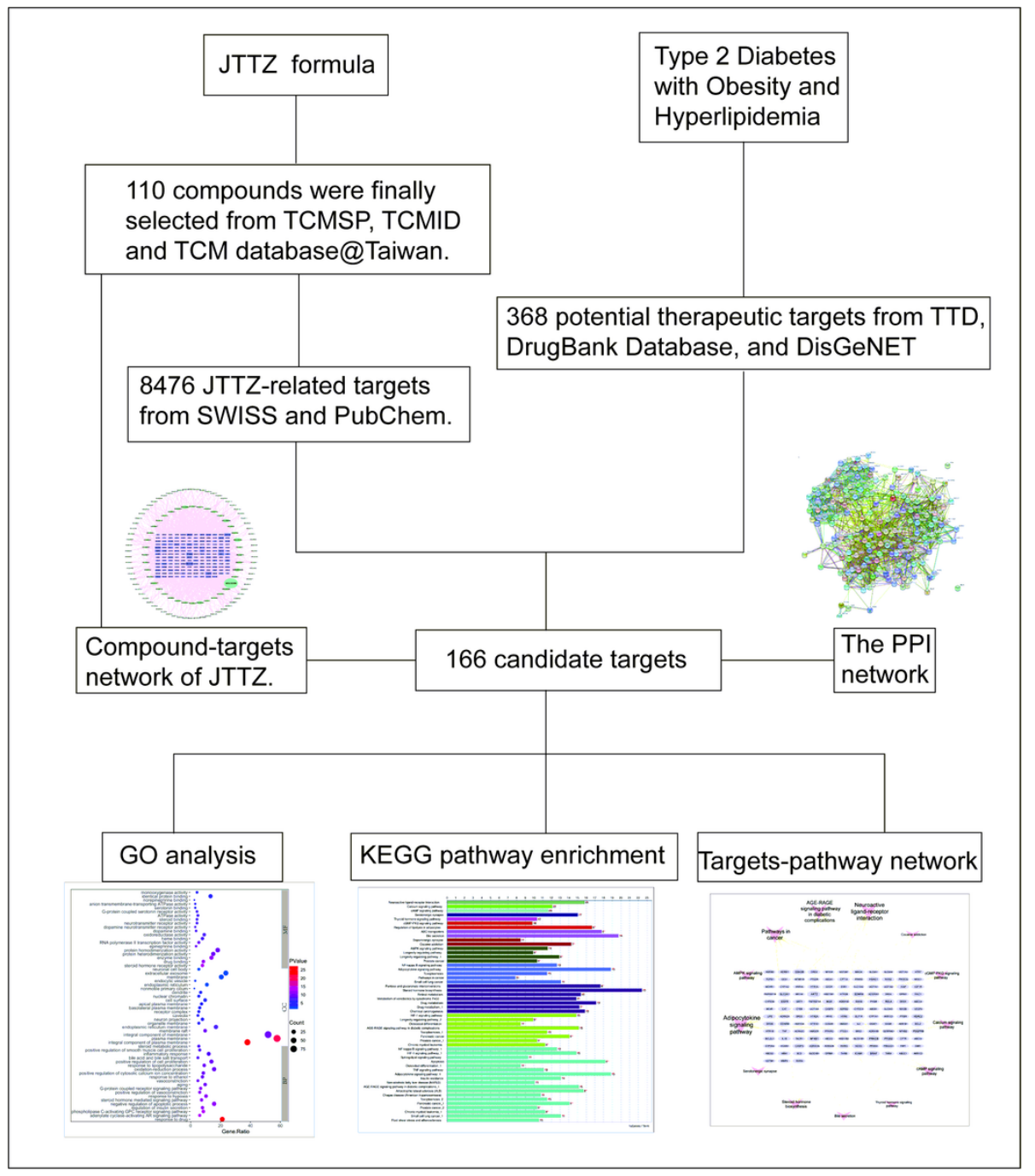

\section{Figure 1}

The flowchart of the network pharmacology for deciphering the mechanisms of JTTZ against T2D with obesity and hyperlipidemia. 


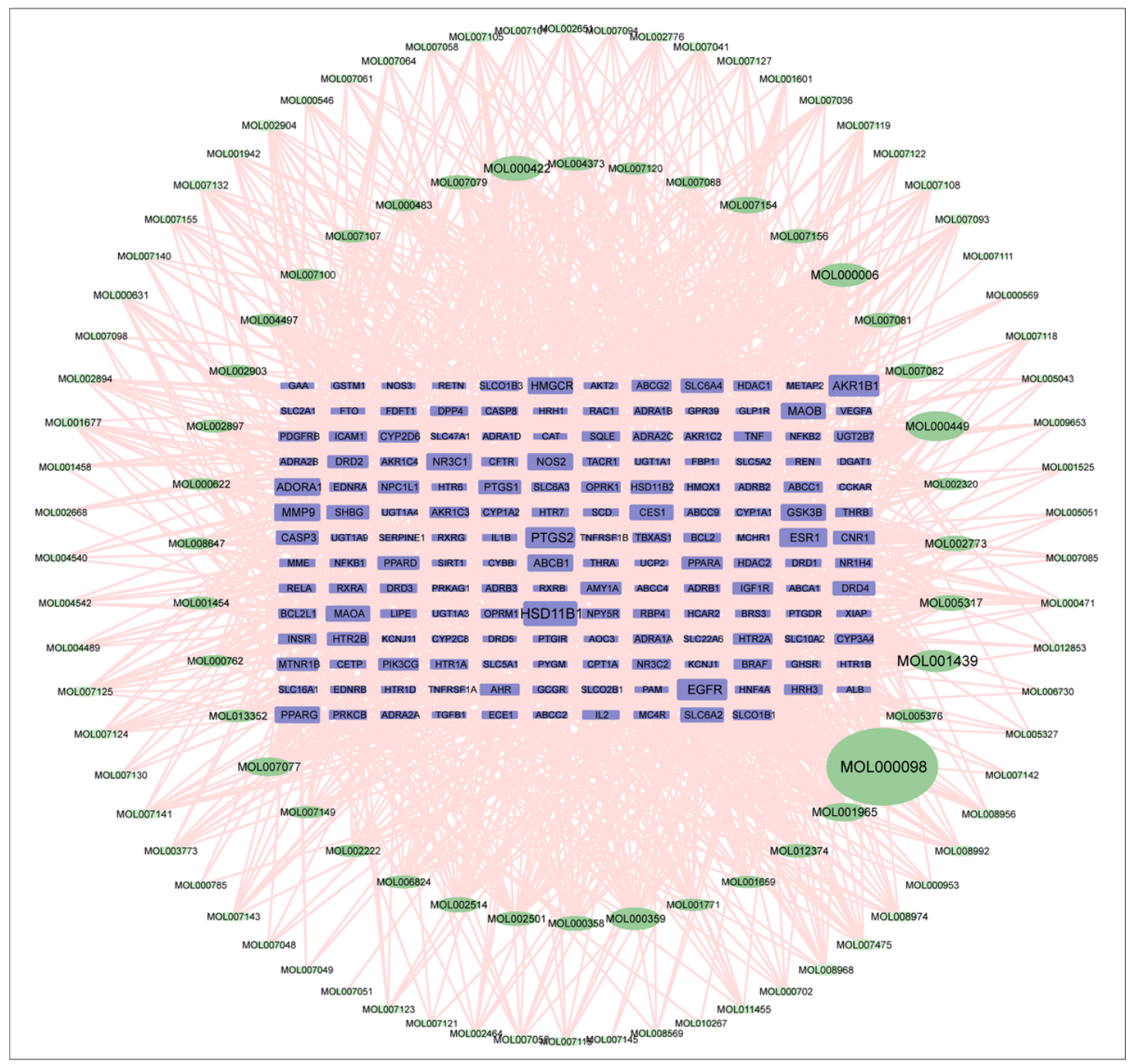

Figure 2

Compound-targets network of JTTZ.

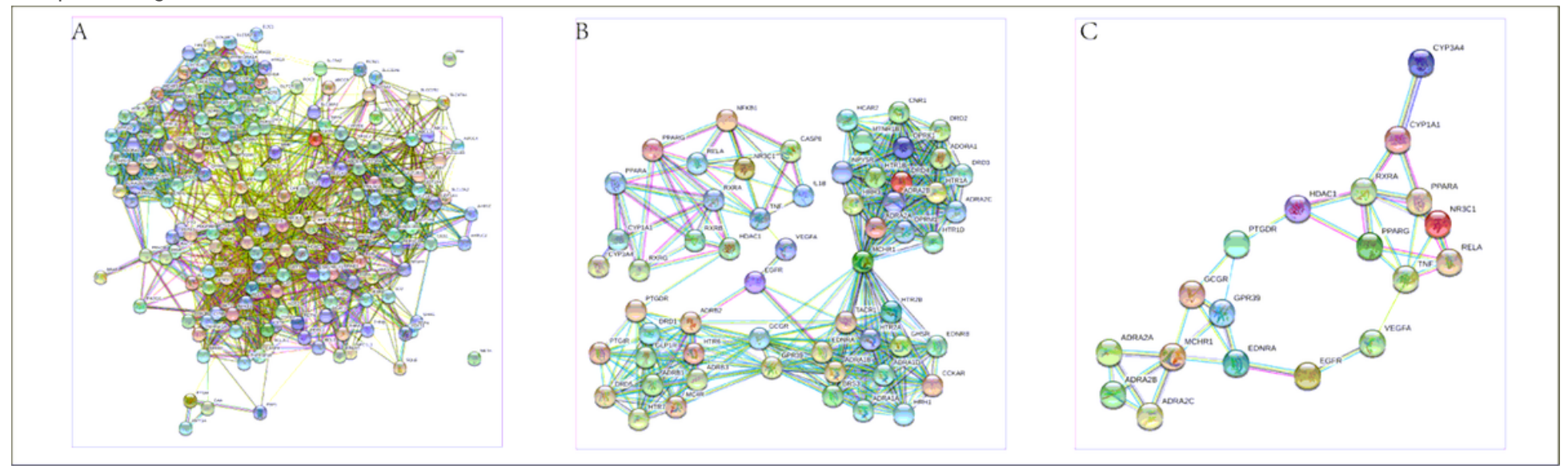

Figure 3

The PPI network of candidate targets for JTTZ against T2D with obesity and hyperlipidemia. 


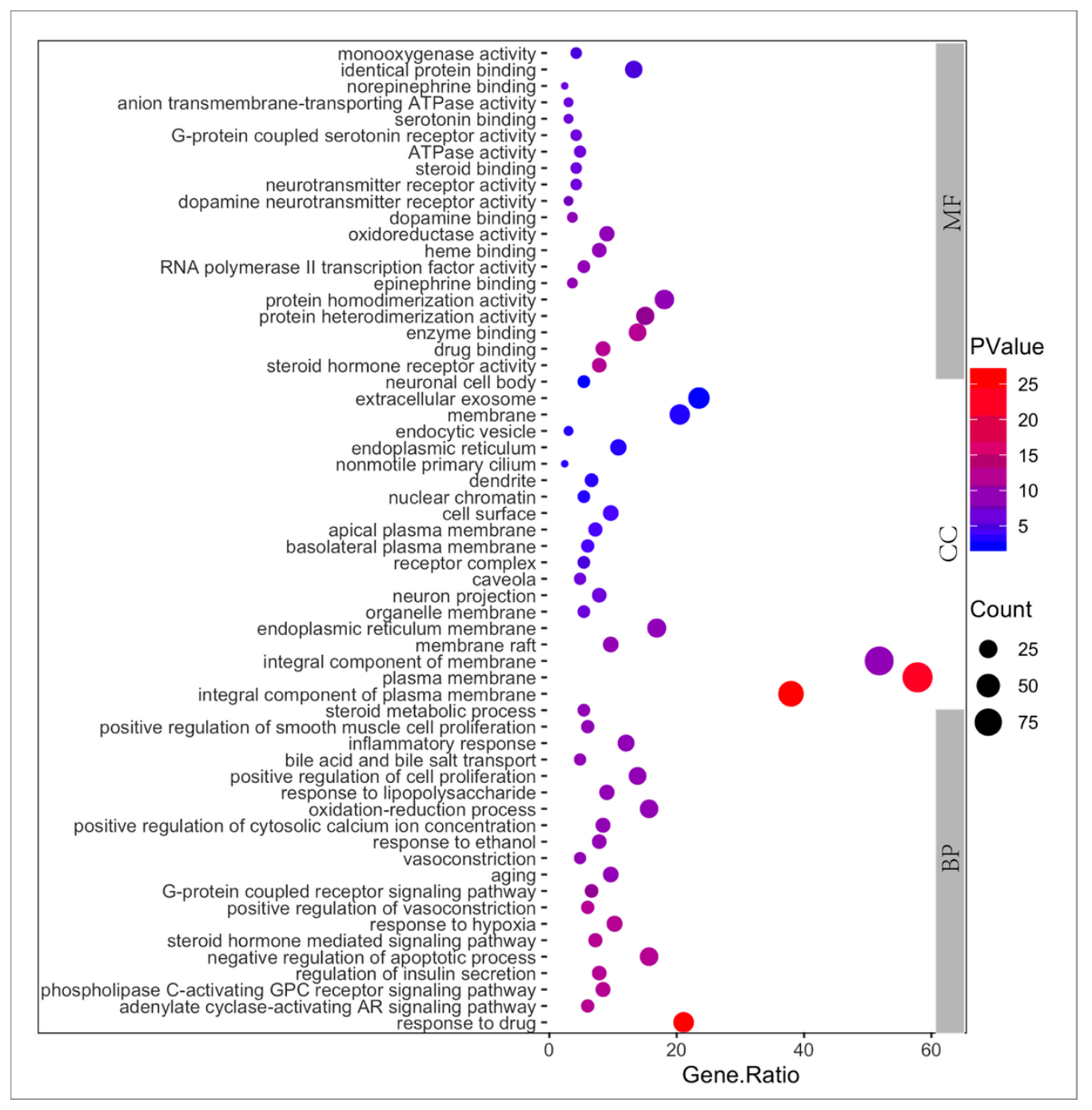

Figure 4

GO analysis of candidate targets of JTTZ against T2D with obesity and hyperlipidemia. 


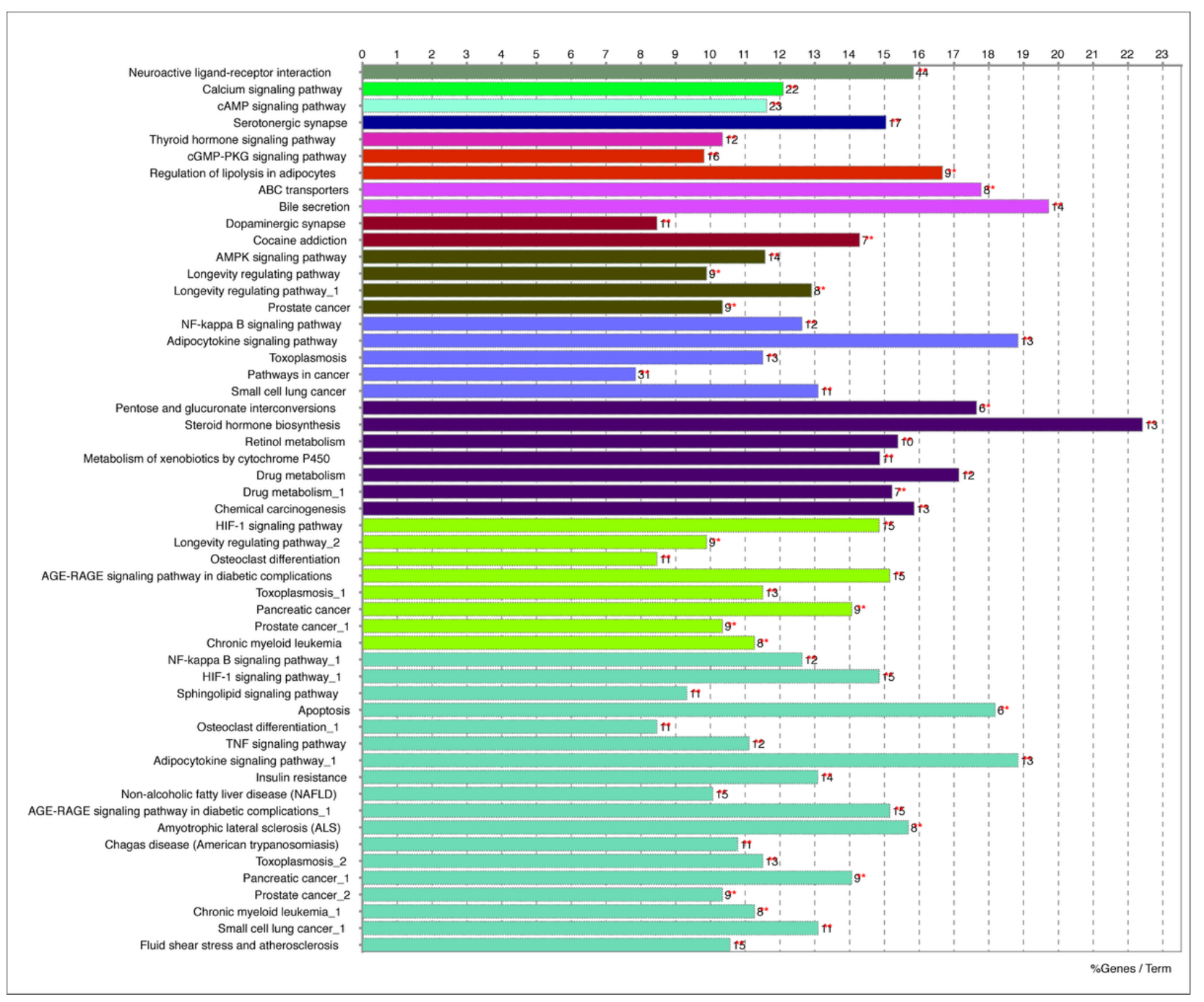

\section{Figure 5}

KEGG pathway enrichment of candidate targets of JTTZ against T2D with obesity and hyperlipidemia. 


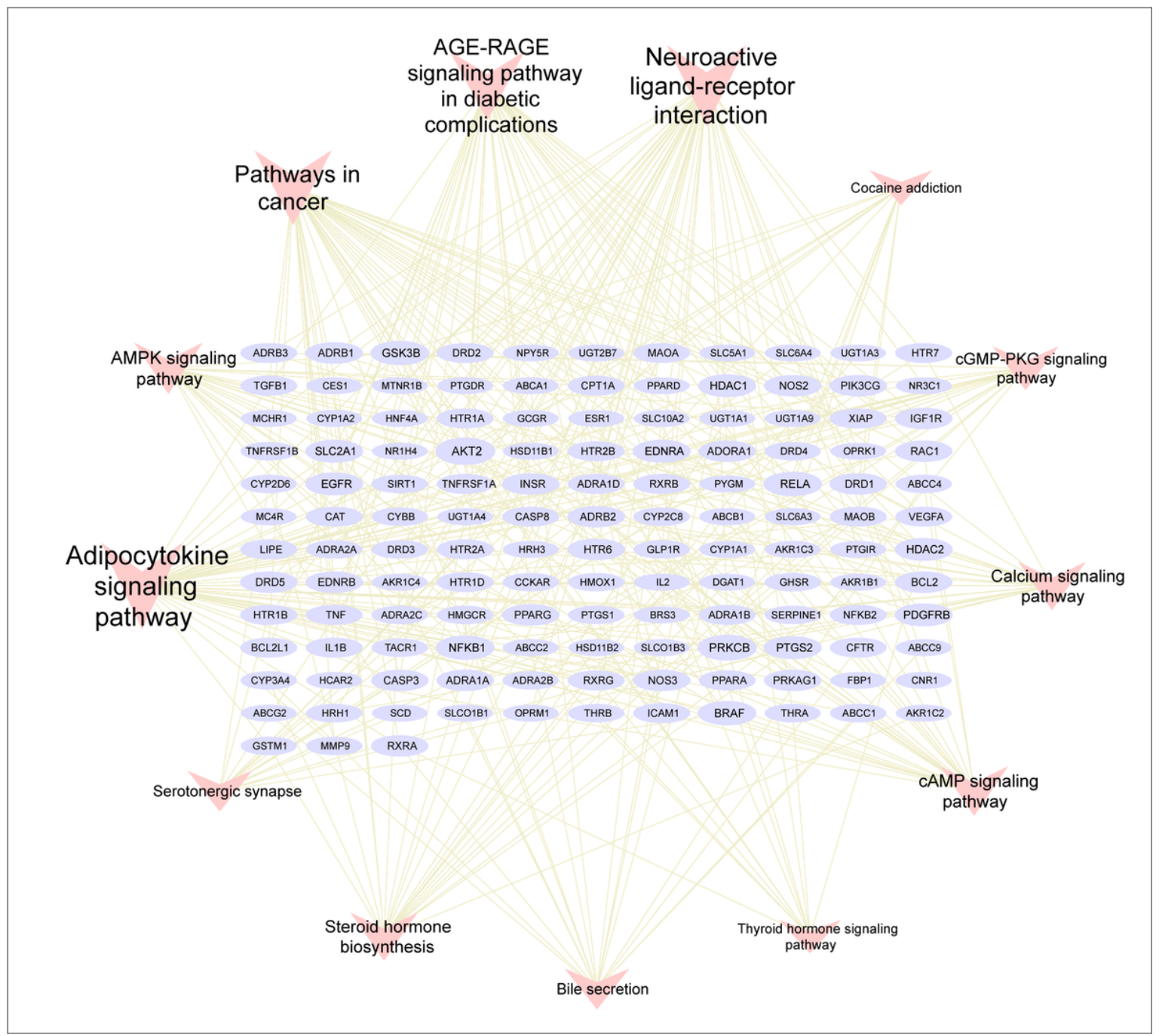

\section{Figure 6}

Targets gene-pathway network of JTTZ against T2D with obesity and hyperlipidemia. 

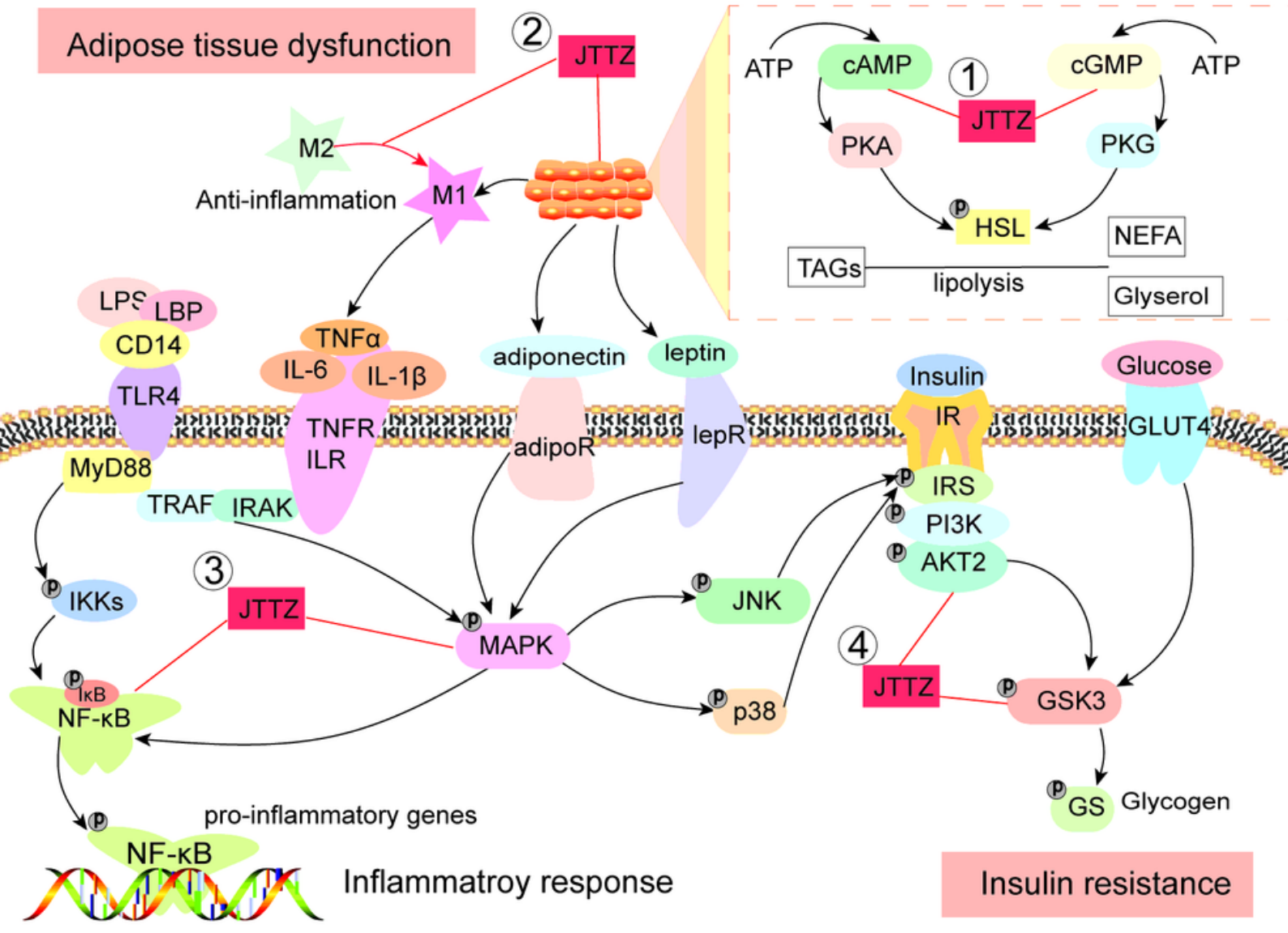

Figure 7

The potential mechanisms of JTTZ against T2D with obesity and hyperlipidemia.

\section{Supplementary Files}

This is a list of supplementary files associated with this preprint. Click to download.

- Supplementarytable1.xlsx

- Supplementarytable2.xIsx 